\title{
Programa de Pós-graduação em Modelos de Decisão e Saúde: uma experiência exitosa na formação em Saúde no Estado da Paraíba.
}

\author{
Program Postgraduate Decision models and Models \\ Health: a successful experience in training in Health \\ in the state of Paraiba.
}

Programa de Pós Grado em Modelos de Decision y Salud: una exitosa experiencia en la formación en Salud en el estado de Paraiba.

\author{
Ronei Marcos de Moraes ${ }^{1}$ \\ Rodrigo Toledo Vianna ${ }^{2}$ \\ Rackynelly Alves Sarmento Soares ${ }^{3}$ \\ César Cavalcanti da Silva ${ }^{4}$
}

RESUMO: Este relato de experiência, entre outros aspectos, apresenta o histórico, a contextualização, os objetivos, as bases teórico-pedagógicas e as estratégias para manutenção do caráter interdisciplinar do Programa de Pós-Graduação em Modelos de Decisão e Saúde. Esse Programa foi desenvolvido pela Universidade Federal da Paraíba (UFPB) no âmbito dos Centros de Ciências da Saúde e Ciencias Exatas e da Natureza, trata-se de uma proposta inovadora de formação interdisciplinar, composto pelas áreas de Ciências Exatas e Ciências da Saúde, com a participação de docentes dos Departamentos Estatística, Informática, Nutrição, Enfermagem em Saúde Pública e Psiquiatria, Enfermagem Clínica, Fisioterapia, Fonoaudiologia, Odontologia Social, Farmácia e Psicologia. A proposta do curso foi aprovado pela CAPES em 2008; o processo de seleção e o início das aulas foram concretizados no primeiro semestre de 2009. Mais do que um processo de capacitação, a proposta inova por seu caráter interdisciplinar. Seu sucesso pode ser observado pelo aumento da demanda nos processos seletivos do Programa, pelo contínuo aumento de produção científica, ampliação de parcerias e sanduíches dos alunos de doutorado em

\footnotetext{
1 Departamento de Estatística, Universidade Federal da Paraíba, João Pessoa-PB, Brasil. E-mail: ronei@de.ufpb.br

2 Departamento de Nutrição, Universidade Federal da Paraíba, João Pessoa-PB, Brasil. E-mail: vianna_rodrigo@ yahoo.com

3 Programa de Pós-graduação em Modelos de Decisão e Saúde, Universidade Federal da Paraíba, João Pessoa-PB, Brasil. E-mail: rackynelly@unb.br

4 Departamento de Enfermagem, Universidade Federal da Paraíba, João Pessoa-PB, Brasil. E-mail: rasecprof@ gmail.com
} 
instituições internacionais e, principalmente, pela alocação dos recursos humanos formados na docência, pesquisa e gestão.

Palavras-chave: Educação de Pós-Graduação. Modelos Estatísticos. Técnicas de Apoio para a Decisão. Tomada de Decisões. Gestão em Saúde.

ABSTRACT: This experience report presents the history, context, objectives, theoretical and pedagogical bases and strategies for maintaining the interdisciplinary nature of the Graduate Program in Decision Models and Health, among other aspects. This Program was developed by Federal University of Paraíba (UFPB) within the Centers of Health Sciences and Exact and Natural Sciences. It is an innovative proposal for interdisciplinary training, with the participation of researchers of Departments of Statistics, Computer Science, Nutrition, Public Health Nursing and Psychiatry, Clinical Nursing, Physical Therapy, Speech Therapy, Social Dentistry, Pharmacy and Psychology. The purpose of that Program was approved by Coordination for the Improvement of Higher Education Personnel (CAPES) in 2008. The first selection process and the start of classes were implemented in the first trimester of 2009. More than a training process, the proposal breaks new ground by its interdisciplinary characteristic. The success achieved can be measured by the increase in demand in the Program selection process, by continuous increase in scientific production, by expanding partnerships and by sandwiches Doctorate students on international institutions and particularly the allocation of human resources trained in teaching, research and health management. Keywords: Education, Graduate. Models, Statistical. Decision Support Techniques. Decision Making. Health Management.

RESUMEN: Este relato, trata especialmente del histórico, del contexto em que se presentaba, los objectivos, las basis teórico-pedagogicas y las estratégias para mantener el caracter interdisciplinário del Programa de Pós Grado em Modelos de Decision y Salud. Este Programa foi desarollado em la Universidad Federal de Paraíba (UFPB) com la participacción de los Centros de Ciencias de la Salud y de las Ciencias Exactas y de la Naturaleza. És uma propuesta inovadora de formacción de recursos humanos com habilidade interdisciplinária em los campos de la salud y de las ciências exatas, donde participan docentes de los departamentos de Estadística, Informática, Nutricción, Enfermaje, Fisioterápia, Fonoaudiologia, Odontologia, Farmácia y Psicolgia. La propuesta del curso de maestria se fue aprobada por CAPES en 2008 y la primera turma ingresó en 2009. Mas que la capacitacción, se intenta inovar por su caráter interdisciplinário. Desde su criacción, há logrado exito que se puede comprovar por el gran numero de personas que procuran el Programa, por lo cresciente numero de publicacciones científicas, por el fortalecimento de la rede de colaboracción internacional y, sobretodo, por el suceso de los egressos ocupando cargos de docência, investigacción e dirección em entidades publicas y privadas.

Palabras clave: Educación de Posgrado. Modelos Estadísticos. Técnicas de Apoyo para la Decisión. Toma de Decisiones.Gestión en Salud. 


\section{Histórico e Contextualização do Programa}

A criação do Programa de Pós-Graduação em Modelos de Decisão e Saúde (PPGMDS) foi consequência da parceria bem sucedida, iniciada em meados de 2004, entre os Departamentos de Estatística, Informática, Nutrição e Enfermagem da Universidade Federal da Paraíba (UFPB) em vários projetos de pesquisa muitos dos quais envolvendo outras instituições de ensino, pesquisa e órgãos públicos ${ }^{5}$. Essa aproximação, saúde-exatas, aconteceu de forma natural motivados pela conciliação de interesses comuns e convergentes: se de um lado, a área da saúde necessitava de técnicas e métodos de análise de dados dos sistemas de informação com enfoque epidemiológico, avaliação das condições de saúde e risco da população, do outro, a área de exatas carecia de casos práticos para aplicação de seus métodos, além de pessoal qualificado para sua proposição.E $\mathrm{s} t \mathrm{e}$ modelo de parceria, que posteriormente passou a ser repetido diversas vezes no PPGMDS, foi inspirador para a definição dos objetivos principais desse Programa de Pós Graduação.

Constatou-se que no âmbito local, regional e nacional não havia nenhum curso que proporcionasse formação com vistas a atender a uma necessidade atual da sociedade, e especialmente da área da saúde. Essa necessidade era confirmada pela busca constante de alunos de cursos de pós graduação da área da saúde para apoio em planejamentos e análises estatísticas mais sofisticadas junto aos docentes do Departamento de Estatística da Universidade Federal da Paraíba.

Deste processo resultou a construção de um grande projeto proposto pelo Departamento de Nutrição, com a participação do Departamento de Estatística, que foi um inquérito de saúde no município de João Pessoa em 2007 chamado "Primeiro diagnóstico e intervenção da situação alimentar, nutricional e das doenças não transmissíveis mais prevalentes da população no município de João Pessoa/PB". Deste projeto originou-se outro denominado "Relação entre renda familiar e consumo alimentar habitual de vitamina com ação antioxidante na população infantil: um estudo de base populacional, 2008-2010".

Todo esse esforço confirmou a necessidade e, em seguida, apontou para a viabilidade da proposição de um programa de pós-graduação com a finalidade de formar profissionais qualificados para suprir uma importante lacuna do conhecimento: profissionais com habilidade de entender problemas de saúde, capazes de realizar análises estatísticas sofisticadas construindo modelos mais próximos da realidade e ainda com a possibilidade de propor modelos voltados para a tomada de decisão em saúde.

Esta habilidade, que anteriormente somente era atingida na constituição de equipes multidisciplinares, deveria ser atingida com formação de profissionais com perfil interdisciplinar.

5 Projeto Modelagem e predição dos casos de Dengue na Paraíba, envolvendo Departamentos de Estatística, Nutrição da UFPB, USP-São Carlos, CNPq.CYBERMED, projeto... envolvendo tais instituições. Análise Espacial para Avaliação da Atenção Básica à Saúde do Município de João Pessoa/PB usando Bases Integradas de Dados, 2006-2008, envolvendo a Secretaria Municipal de Saúde de João Pessoa 
Diante disso, foi proposto o Programa de Pós Graduação Modelos de Decisão e Saúde, sendo um programa interdisciplinar que congrega as áreas das exatas e as áreas da saúde.

Assim, no ano de 2008, foi aprovado pela CAPES o PPGMDS em nível de mestrado. No final deste mesmo ano foi feito o primeiro processo seletivo com o ingresso dos primeiros alunos selecionados, em março de 2009. Já em 2011 o curso de Doutorado foi aprovado pela CAPES, tendo a primeira turma ingressada no início de 2012, sendo que os primeiros doutores se titularam em 2015. Desde então, a alta demanda verificada ano a ano nos processos seletivos do PPGMDS, só confirma a necessidade de formação nessa área de conhecimento (Tabela 1).

Tabela 1. Evolução do número de candidatos e aprovados no PPGMDS, desde a sua criação

$\begin{array}{lccc}\text { Ano } & \text { Nível } & \text { Candidatos inscritos }- & \text { Total matriculados } \\ 2009 & \text { Mestrado } & 83 & 16 \\ 2010 & \text { Mestrado } & 93 & 17 \\ 2011 & \text { Mestrado } & 107 & 18 \\ 2012 & \text { Mestrado } & 160 & 12 \\ 2012 & \text { Doutorado } & 28 & 05 \\ 2013 & \text { Mestrado } & 91 & 20 \\ 2013 & \text { Doutorado } & 35 & 05 \\ 2014 & \text { Mestrado } & 80 & 22 \\ 2014 & \text { Doutorado } & 37 & 16 \\ 2015 & \text { Mestrado } & 74 & 21 \\ 2015 & \text { Doutorado } & 37 & 16\end{array}$

Figura 1 - Linha do tempo da contextualização histórica do PPGMDS (2004-2015)

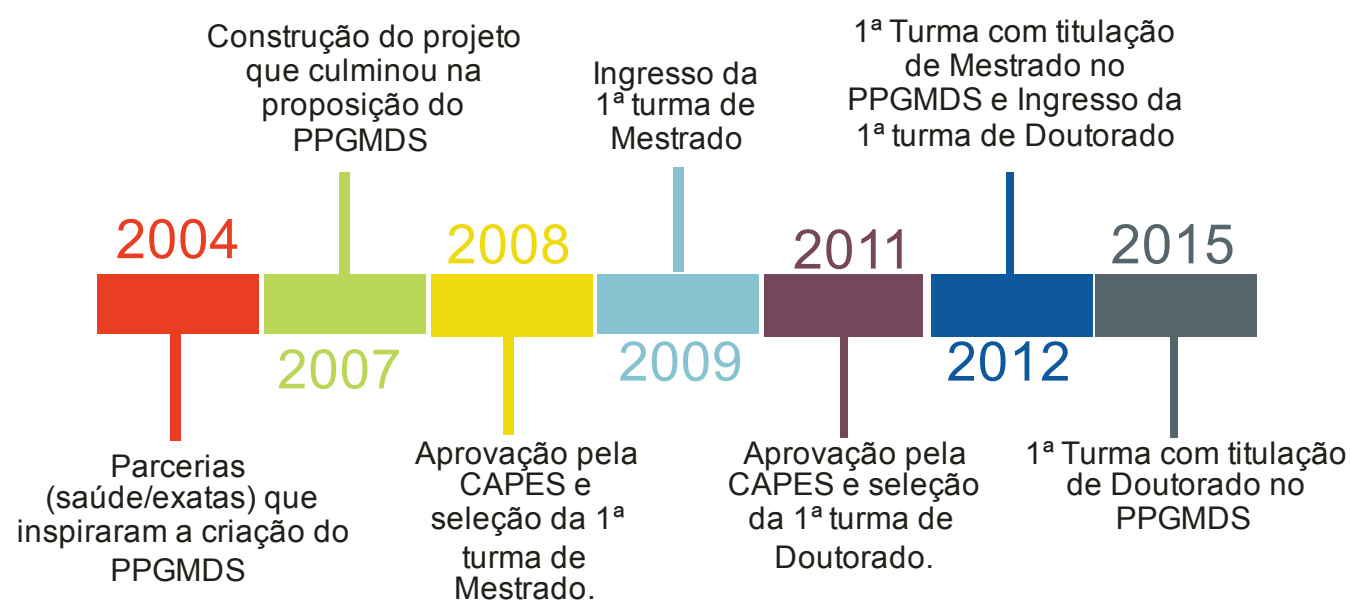

Fonte: Elaboração própria.

\section{Pressupostos teóricos que fundamentam a concepção pedagógica do curso}

Pedagogicamente, todos os Programa de Pós-graduação perseguem as orientações propostas na Lei 9.394 de 17 de dezembro de 1996, especialmente, os Princípios e Fins da Educação Nacional e seus Artigos $2^{\circ} 3^{\circ}$ 
A educação, dever da família e do Estado, inspirada nos princípios de liberdade e nos ideais de solidariedade humana, tem por finalidade o pleno desenvolvimento do educando, seu preparo para o exercício da cidadania e sua qualificação para o trabalho $\square$ Artigo $2^{\circ}$ da Lei 9.394/96.

O ensino será ministrado com base nos seguintes princípios:

I Pluralismo de ideias e de concepções pedagógicas $\square$ Artigo $3^{\circ}$ Inciso III

Classificado como Interdisciplinar pelas instituições de fomento brasileiras, o Programa de Pós-graduação em Modelos de Decisão e Saúde, prima pela pluralidade de ideias e concepções pedagógicas. Com a disciplina Metodologia do Ensino Superior há um cuidado especial com a formação didática de seus egressos.

A disciplina em lide, atualiza os discentes do Programa sobre os conteúdos relativos ao processo ensino-aprendizagem e manuseio do ferramental específico para os processos de regência de aulas, em nível superior, de modo a criar no mestrando ou doutorando uma postura crítica, reflexiva e questionadora frente aos processos pedagógicos de formação de força de trabalho.

Essa formação, compreende a reflexão e compreensão do processo de planejamento educacional e de planejamento de ensino, no contexto da formação de força de trabalho; Reflete sobre as abordagens pedagógicas utilizadas no ensino para a formação de força de trabalho nas ciências exatas e nas ciências da saúde, com ênfase nas classificações de autores contemporâneos, além de refletir sobre a inserção de novas abordagens pedagógicas no processo de ensino para a formação da força de trabalho, com ênfase na Abordagem por Competências.

\section{Descrição do PPGMDS}

Trata-se de um programa interdisciplinar composto pelas áreas de Ciências Exatas e Ciências da Saúde, com a participação de docentes dos Departamentos Estatística, Informática, Nutrição, Enfermagem em Saúde Pública e Psiquiatria, Enfermagem Clínica, Fisioterapia, Fonoaudiologia, Odontologia Social, Farmácia e Psicologia. Sua área de concentração é em Modelos de Decisão e Saúde com duas linhas de pesquisa, Modelos em Saúde e Modelos de Decisão. Trata-se portanto de uma ação estratégica para o desenvolvimento do ensino, formação, pesquisa e gestão nas áreas de modelagem e tomada de decisão e saúde sob a ótica interdisciplinar.

A linha de pesquisa Modelos em Saúde visa a realização de estudo de casos e situações na área de Saúde Pública, nas quais há a necessidade de tomada de decisão sobre informações e/ou dados, sejam eles qualitativos ou quantitativos. Pesquisa e utilização de modelos para o gerenciamento, análise e tomada de decisão sobre informações oriundas de processos de Saúde Pública.

A linha de pesquisa Modelos de Decisão envolve pesquisa sobre o processamento de informações em várias áreas da Saúde, abrangendo o uso de modelos matemáticos, estatísticos 
e computacionalmente inteligentes sob uma ótica de suporte a tomada de decisão. Deste modo, esta linha desenvolve aplicações que se fundamentam nesses modelos, estimulando a investigação interdisciplinar.

Assim, com base nessas linhas de pesquisa o objetivo geral do PPGMDS é formar recursos humanos voltados para a compreensão e atuação dentro da realidade brasileira, seja no âmbito local, regional ou nacional, diferenciados e qualificados com domínio de conhecimento das Ciências Exatas e das Ciências da Saúde, independente das suas formações básicas, estando aptos para a docência, pesquisa e gestão com capacidade para tomada de decisões com embasamento teórico específico e com propriedade de conhecimento sobre o problema.

Até 2013 o PPGMDS a página Web era www.de.ufpb.br/ mds e a partir de 2014, a UFPB sugeriu a unificação das páginas Web no formato SIGAA (http://www.ufpb.br/pos/mds). Mesmo assim, as páginas antigas do PPGMDS continuam disponíveis a título de histórico e para manter as versões finais das dissertações defendidas desde o início das suas atividades até o ano de 2013.

\section{Estratégias para manutenção do caráter interdisciplinar do PPGMDS}

A formação interdisciplinar do colegiado do PPGMDS, composto inicialmente por docentes dos Departamentos de Estatística, Computação, Nutrição, Odontologia Social, Enfermagem em Saúde Coletiva e Enfermagem Clínica é um elemento importante no fortalecimento do caráter interdisciplinar do programa. Inicialmente, o Programa contava com apenas 11 docentes, sendo dois deles colaboradores. Ainda assim, apesar do número reduzido de docentes foi possível criar as condições fundamentais para existência do programa e estruturar as bases para seu crescimento. Atualmente, o Programa conta com 20 docentes em seus quadros.

O crescimento do PPGMDS traduzido em aumento do número de trabalhos, de projetos, da procura por vagas, tornou oportuna a inclusão de novos docentes. Desta forma, através de um processo de credenciamento, regulamentado por Resolução interna do PPGMDS, foram aprovados novos docentes dos Departamentos de Fisioterapia, Fonoaudiologia, Farmácia e Psicologia, aumentando também a possibilidade de realização de projetos nestas novas áreas do conhecimento, como também receber alunos de mais variada formação.

Outra estratégia adotada para a manutenção e fortalecimento do caráter interdisciplinar do programa refere-se a dupla orientação de cada todos discentes, seja do nível de mestrado, seja do doutorado por dois docentes, sendo obrigatoriamente um das Ciências Exatas e outro das Ciências da Saúde. Quando necessário, ainda é possível agregar um co-orientador, interno ou externo ao Programa. Esta decisão do PPGMDS tem se mostrado um diferencial importante que garante a formação interdisciplinar do aluno e induz o desenvolvimento de projetos com maior integração do corpo docente. Todos os docentes do programa passam a realizar atividades com enfoque interdisciplinar, preenchendo uma lacuna do conhecimento na área das ciências exatas aplicadas (modelos de decisão em saúde) bem como na área das ciências da saúde (modelos de saúde) com trabalhos de alto nível e formando profissionais habilitados para atuarem e resolverem problemas 
complexos e interdisciplinares.

Essa experiência multidisciplinar é muito rica para o conjunto de alunos de cada turma, ao longo dos cursos. Por serem oriundos de cursos das Ciências Exatas e da Natureza, das Ciências da Saúde, das Engenharias e Psicologia, as turmas são bastante heterogêneas e a discussão viva das habilidades de cada discente tem produzido um caminho muito exitoso para se romper as barreiras das disciplinas e formar profissionais com capacidades e habilidades interdisciplinares.

Por esses fatos, o PPGMDS já é reconhecido como um programa inovador e que tem obtido resultados positivos na formação de profissionais bastante demandados pela academia e pelos serviços de saúde, especialmente nas áreas estratégicas de planejamento nos três níveis de governo, suprindo uma carência histórica seja de profissionais de exatas com pleno conhecimento e compreensão dos problemas de saúde ou profissionais de saúde com habilidades avançadas de análise e modelagem estatística e tomada de decisão.

\section{Perfil do Egresso}

O perfil do egresso do PPGMDS consiste em um profissional com formação interdisciplinar, com capacidade para o melhor entendimento dos fenômenos da área da saúde e suas interrelações, considerando sua complexidade e emergência e com habilidade para realizar abordagens estatísticas e computacionais mais complexas do que aquelas usualmente utilizadas na área, tanto para a descrição dos fenômenos de saúde, predição de eventos como representações das múltiplas relações existentes entre os eventos de saúde e doença e seus condicionantes. Estas características permitem ao tomador de decisões melhores possibilidades de acertos nos encaminhamentos de ações, programas e políticas no campo da saúde, tanto tratando de indivíduos como de coletividades.

Desta forma os egressos do PPGMDS têm grande potencial de inserção no mercado de trabalho, executando funções administrativas em órgão públicos ligados ao cuidado da saúde de populações, como as secretarias de saúde e órgãos gestores dos três níveis de governo. De forma análoga, devido sua formação interdisciplinar, também são fortes candidatos ao ingresso nas instituições de ensino superior, especialmente as Universidades Federais, Estaduais e Particulares, como docentes ou em cargos técnicos, desenvolvendo atividades de ensino, pesquisa e extensão com a finalidade de formar novos e qualificados recursos humanos.

O PPGMDS também tem formado candidatos para o curso de doutorado de outros Programas de Pós Graduação em vários Estados do País. Nestes cinco anos de funcionamento do PPGMDS, praticamente todos os egressos do curso de mestrado e de doutorado estão atuando nestas áreas descritas acima, com poucas exceções atuando em empreendimentos próprios.

Em relação a permanência do corpo discente no programa, o tempo médio de defesa de doutorados pelo PPMGDS é atualmente de 43,67 meses e de mestrado é de 23,75 meses (Tabela 2). Vale ressaltar a preocupação do Programa com a qualidade dos examinadores externos presentes nas bancas de avaliação, sendo que em várias delas, os membros externos são bolsistas de Produtividade 
em Pesquisa do CNPq.

Table 2- Distribuição das defesas por ano segundo o nível (Doutorado e Mestrado).

\begin{tabular}{ccc}
\hline Ano de defesa & Nível Mestrado & Nível Doutorado \\
\hline 2010 & 2 & - \\
2011 & 14 & - \\
2012 & 14 & - \\
2013 & 14 & - \\
2014 & 11 & - \\
2015 & 12 & 05 \\
2016 & 12 & 01 \\
Total & 79 & 06 \\
\hline
\end{tabular}

\section{Acompanhamento de Egressos}

De acordo com os objetivos e a característica interdisciplinar proposta pelo PPGMDS, os alunos oriundos do Programa, seja no nível de mestrado ou, com maior propriedade, do doutorado, terão formação sobre temas relacionados a modelagem do conhecimento e tomada de decisões com embasamento teórico específico e com propriedade de conhecimento sobre eles, independentemente da formação básica haver sido dentro das áreas de Ciências Exatas, da Saúde ou das Engenharias.

O destino dos egressos do Programa, são os órgãos competentes de saúde nos três níveis de governo, hospitais ou outras instituições de saúde do setor público ou privado e órgãos gestores, utilizando seus conhecimentos para uma gestão mais eficiente e profissional nas áreas envolvidas. Além disso os egressos podem atuar na área da docência e da pesquisa, visto a formação conceitual aprofundada e diferenciada da qual se apropriaram durante sua formação (Figura 2).

Figura 2 - Atuação do aluno egresso do PPGMDS.

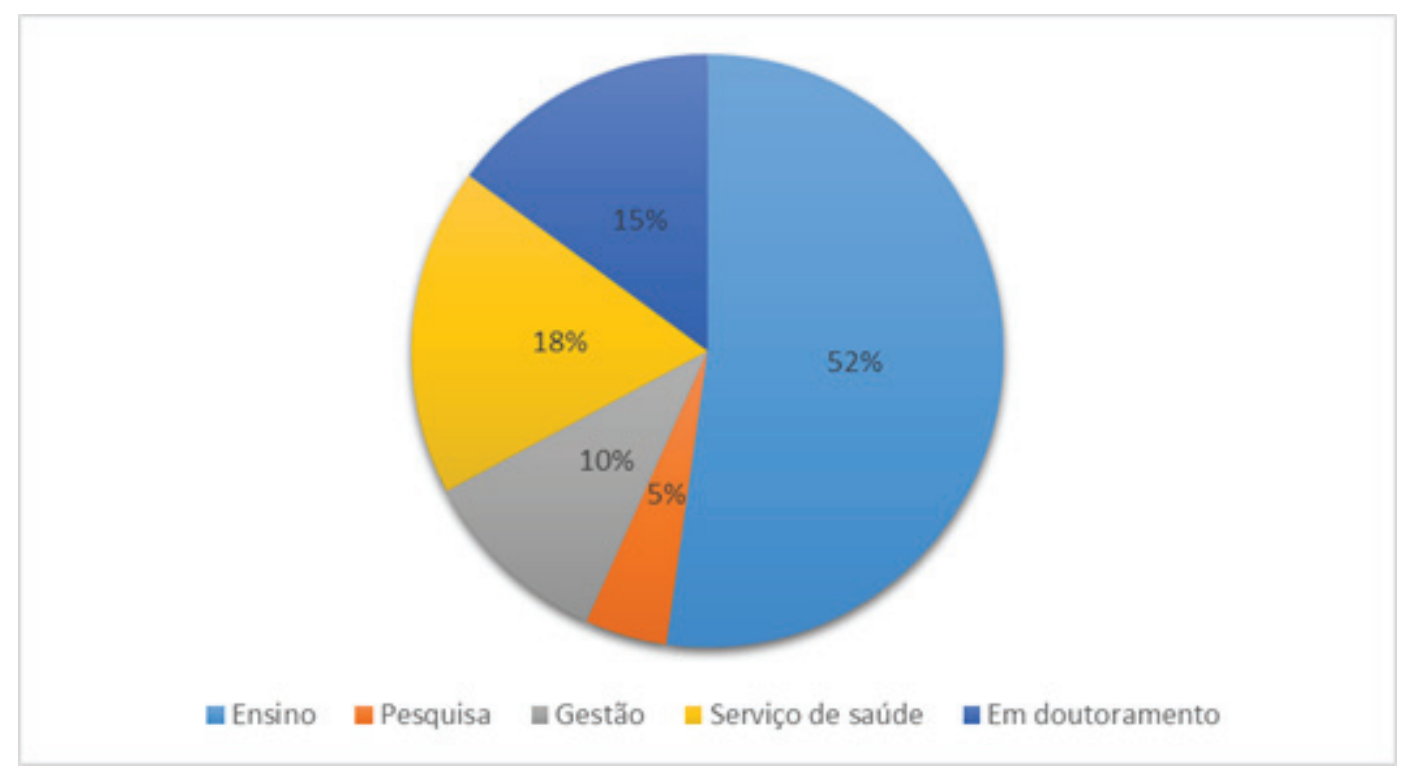




\section{Estrutura Curricular}

Os cursos de mestrado e doutorado do PPGMDS são compostos por disciplinas com contagem de créditos correspondendo cada crédito a 15 horas-aula de atividades teóricas de ensino ou 30 horas-aula de atividades práticas. Para a integralização do curso de Mestrado do PPGMDS são necessários o cumprimento de 29 créditos, sendo 14 créditos obrigatórios (conhecimentos básicos) e 15 optativos (formação específica) que podem ser entre aquelas disciplinas oferecidas pelo PPGMDS ou outros programas de Pós-Graduação reconhecidos pela CAPES. Dentre os créditos obrigatórios encontra-se a atividade acadêmica de Estágio Docência que corresponde a um crédito prático.

No curso de Doutorado, para sua integralização, são necessários 38 créditos, sendo 26 obrigatórios e 12 optativos que, tal como o mestrado, podem ser cursados no próprio PPGMDS como em outros programas de Pós-Graduação. No curso de Doutorado faz-se necessário o cumprimento de dois créditos práticos na atividade acadêmica de Estágio Docência.

Os conhecimentos básicos para o Mestrado dizem respeito à metodologia epidemiológica, álgebra computacional, probabilidade e estatística e o estudo das formas de modelar dados e informações de modo a possibilitar tomar decisões. A disciplina "Projeto de Pesquisa de Mestrado", com 3 créditos práticos, tem como finalidade auxiliar na construção do projeto de dissertação do discente e corresponde a uma carga horária de 90 horas. Esta formação é complementada com uma atividade de "Seminários em Modelos de Decisão e Saúde" que consiste na participação dos discentes em palestras de interesse geral e que são geralmente ministradas por convidados externos ao PPGMDS e que corresponde a um crédito teórico.

No caso do Doutorado, essas mesmas disciplinas são acrescidas de uma disciplina teórica "Filosofia da Ciência" com a finalidade apresentar para os futuros pesquisadores aspectos dos processos e práticas da pesquisa e da construção do conhecimento científico, de modo a estimular o desenvolvimento e potencialidades da área interdisciplinar em que o Programa esta inserido. Também são propostas duas disciplinas práticas denominadas "Projeto de Doutorado I e II" orientadas para construção do projeto de tese, com 4 créditos práticos, correspondendo a uma carga horária de 120 horas cada.

A formação específica das disciplinas optativas é definida de acordo com a linha de pesquisa do aluno e um elenco de 22 disciplinas são oferecidas para o mestrado e 29 para o Doutorado. Entre estas disciplinas se recomenda fortemente que o aluno curse a disciplina "Metodologia do Ensino Superior", atendendo a um compromisso do programa em capacitar o pós-graduando com relação ao binômio ensino-pesquisa. Entre as optativas estão disciplinas avançadas de modelos de decisão, análise estatística, métodos computacionais, políticas de saúde e epidemiologia. Ainda existem opções para estudos e pesquisas específicas, individuais ou coletivas, através das disciplinas "Estudos Especiais" e “Tópicos Especiais". A disciplina denominada "Estudos Especiais" são de 
caráter individal e devem versar exclusivamente sobre assunto relacionado com o tema do trabalho final do discente. A disciplina de Tópicos Especiais são disciplinas de caráter coletivo e possuem duas variações: Tópicos Especiais em Decisão e Tópicos Especiais em Saúde. Ambas são disciplinas com ementa variável e devem abranger conteúdos de fronteira no estado da arte da especialidade a que se refere e/ou complementar ou expandir a formação científica do aluno na linha de modelos de decisão ou de saúde. O discente poderá cursar essas duas disciplinas mais de uma vez desde que se aborde conteúdos diferentes em cada uma delas, até o limite máximo de 06 (seis) créditos. Tanto a disciplina de "Estudos Especiais" quanto as de "Tópicos Especiais" devem ter seus conteúdos aprovados previamente pelo Colegiado do PPGMDS para serem ofertadas.

\section{Estágio de docência}

O Estágio de Docência é obrigatório a todos os alunos do Programa. É regido por norma específica da UFPB através da Resolução 26/1999 e está limitado a um semestre letivo para alunos de mestrado, não podendo ultrapassar 30 horas aula e dois semestres letivos para alunos de doutorado, que da mesma forma, não pode ultrapassar 30 horas aula por semestre.

Todos os alunos do Programa realizam seu estágio docência no ano posterior à sua entrada. A estrutura curricular do programa estabelece que o aluno deve realizar a atividade de estágio docência depois de apresentar e ter seu projeto de pesquisa aprovado no exame de qualificação. Esta atividade tem se mostrado muito produtiva tanto para a formação do aluno, do ponto de vista do conhecimento de técnicas pedagógicas e experiencias em avaliação de alunos, como também para a transformação necessária do mestrando-aluno ou doutorando-aluno em mestre-professor ou doutor-professor, respectivamente. Sendo esta uma oportunidade singular para aperfeiçoamento de suas técnicas pedagógicas muitas das quais vistas na disciplina optativa oferecida pelo programa, denominada "Metodologia do Ensino Superior", e fortemente recomendada que seja cursada por todos os discentes, favorece muito o sucesso desta atividade.

Observamos nesta prática que os mestrandos e doutorandos, na posição assistida de professores, são defrontados com a necessidade de passar os seus conhecimentos para os alunos de graduação e este desafio consegue, em muitos casos, mudar a postura do pós graduando, comprometendo-o mais fortemente com a missão escolhida por ele de seguir a carreira de ensino e pesquisa.

\section{Infraestrutura}

A infraestrutura laboratorial do PPGMDS está dividida em três segmentos: laboratórios de pesquisas, laboratórios de uso geral, incluindo um exclusivo para alunos do PPGMDS e laboratórios didáticos (para uso uso em aulas); sendo que todos eles são laboratórios computacionais.

O Laboratório de Estatística Aplicada ao Processamento Digital de Imagens e Geoprocessamento (LEAPIG) se destina a pesquisa em métodos de classificação de imagens multiespectrais de sensoriamento remoto e métodos de modelagem estatística e análise de dados georeferenciados de 
Saúde Pública.O Laboratório de Estudos Demográficos (LED) se destina a pesquisa e modelagem de dados demográficos. Possui quatro computadores de uso geral com nobreaks, uma impressora matricial e uma jato de tinta. O Laboratório de Tecnologias para o Ensino Virtual e Estatística (LabTEVE) destina-se a pesquisa de tecnologias para suporte ao ensino virtual nas áreas de estatística e biomédica e desenvolvimento de metodologias de avaliação estatística do aprendizado em sistemas de treinamento na área de saúde.

Em relação ao LabTEVE, no final do ano de 2014, iniciou-se a instalação da primeira CAVE reconfigurável do Nordeste brasileiro (com possibilidade de uso de 1, 2 ou 3 telas de projeção, sendo que essa configuração é adaptável à escolha do usuário) e que foi completada no início de 2015 e está operacional.

O PPGMDS possui uma infraestrutura descentralizada de recursos informacionais, distribuída no Centro de Ciências Exatas e da Natureza (CCEN) e no Centro de Ciências da Saúde (CCS). Conta com laboratórios de uso geral, incluindo um exclusivo para alunos do PPGMDS e laboratórios didáticos (para uso uso em aulas).

O PPGMDS tem acesso à infraestrutura descentralizada de bibliotecas da UFPB. De interesse direto, os alunos do programa podem acessar as bibliotecas físicas: Biblioteca Central da UFPB, Biblioteca Setorial do Centro de Ciências da Saúde, Biblioteca Setorial do Centro de Ciências Exatas da Natureza e Biblioteca Setorial do Centro de Informática para consulta e retirada do acervo. Caso necessário, as demais Bibliotecas Setoriais ainda podem ser consultadas. Segundo dados da Biblioteca Central, a UFPB possui disponível mais de 37.000 livros, cerca de 4.500 títulos de periódicos, dos quais 523 são assinaturas de periódicos de referência.

\section{Integração com a graduação}

Há uma participação efetiva dos docentes credenciados no PPGMDS junto à formação dos cursos de graduação (Bacharelados em Estatística, Informática, Nutrição, Enfermagem, Fisioterapia, Odontologia e Psicologia) e mesmo em outras graduações o que favorece a integração com o PPGMDS. Em todos os grupos de pesquisa do PPGMDS acontece a participação de alunos dessas graduações. Essa participação acontece no apoio a projetos de dissertação, de tese, da própria produção intelectual, através de artigos publicados em congressos nacionais e internacionais e em revistas científicas.

Por se tratar de um Programa caracterizado pela realização de pesquisas voltadas para a tomada de decisão em saúde, com a coleta de dados utilizando metodologias qualitativas ou quantitativas sobre processos de Saúde Coletiva, muitos trabalhos envolvem atividades de campo, seja para realizar entrevistas junto a população, seja para coletar dados climáticos ou conhecer sistemas e bancos de dados de órgãos públicos.

Estas atividades são realizadas em várias situações com a participação de estudantes de graduação 
que vivenciam atividades práticas de pesquisa ao mesmo tempo que colaboram com o trabalho que necessita ser executado. Muitos destes alunos formalizam esta atividade por períodos curriculares maiores possibilitando a equivalência em créditos nos seus cursos de graduação ou realizando atividade institucional de iniciação científica, como bolsistas (PIBIC) ou como voluntários (PIVIC), ou ainda como bolsistas de extensão (PROBEX).

Um exemplo desta situação são os projetos “A Linha do cuidado em saúde bucal na primeira macro regional de saúde do Estado da Paraíba" que conta oficialmente com 13 alunos de graduação participando da pesquisa ou o projeto "Estudo de seguimento da situação de segurança alimentar em famílias residentes em domicílios no interior do estado da Paraíba" que contou com a participação de 23 alunos de graduação, dos cursos de nutrição, enfermagem, biologia, engenharia de alimentos e farmácia que receberam bolsas de Iniciação Tecnológica Industrial do CNPq durante parte da vigência do projeto.

Os docentes do PPGMDS também atuam em orientação de iniciação científica e orientaram também trabalhos de conclusão de curso de graduação nos respectivos cursos nos quais estão vinculados. A existência da Pós Graduação, especialmente no caso de um programa interdisciplinar como o PPGMDS, amplia as possibilidades de trabalho e pesquisa dos alunos de graduação.

Outra forma de interação com a graduação também se dá através do Estágio Docência. O discente atua em uma disciplina, voltada para a graduação, se mestrando, ou em duas se doutorando, auxiliando um docente. Sob a supervisor do docente, esse aluno aprende exercitando na prática, como se prepara uma aula, como utilizar os recursos didático-pedagógicos disponíveis, aplicação e correção de atividades e provas, etc.

Desse modo, a interação com a graduação se dá em vários níveis proporcionando ao discente de graduação um vislumbre do que é uma pós graduação. Ele pode participar de pequenos projetos de pesquisa diretamente (bolsas de iniciação científica), integrar projetos maiores com equipes interdisciplinares e obviamente participar das publicações oriundas desses projetos.

A Resolução que aprova o Regulamento e Estrutura Acadêmica do PPGMDS (Resolução 17/2011), vigente em 2015 até o mês de novembro, admitia que, a critério de seu Colegiado que poderão matricular-se alunos de graduação plena como alunos especiais em disciplinas avulsas que totalizem no máximo nove créditos desde que tenham cumprido pelo menos $80 \%$ dos créditos exigidos para a integralização da estrutura acadêmica de seus cursos. Dessa forma, a interação com a graduação que já vinha sendo realizada, passou a ser cumprimento de uma ação regulamentar.

No final de 2015, o PPGMDS atualizou o seu Regimento, através da Resolução 52/2015, de modo a se aperfeiçoar e se adaptar a uma maior demanda de alunos de graduação. Nessa Resolução, o aluno especial poderá cursar um máximo de até $50 \%$ dos créditos necessários para a integralização do curso, em conformidade com o estabelecido pelo regulamento do PPGMDS, 
sendo no máximo metade destes créditos em disciplinas obrigatórias e no máximo metade destes créditos em disciplinas optativas.

\section{Intercâmbios Nacionais}

Os docentes que fazem parte do quadro permanente do Programa de Pós-Graduação em Modelos de Decisão e Saúde já formam uma integração interdisciplinar por si só, dentro da própria instituição. Estes docentes são oriundos das áreas de exatas e saúde, sendo que essa atuação em ambas as áreas originou-se há algum tempo. Esse é o caso da integração com outras Pós-Graduações dentro da própria UFPB e fora dela. Esses pesquisadores vêm mantendo intercâmbios específicos com outras instituições de pesquisa, quer como participantes eventuais de outros programas de Pós-Graduação quer como colaboradores em diversos projetos de pesquisa em andamento ou já finalizados.

Estas interações se fazem com o Laboratório Nacional de Computação Científica (LNCC), Instituto Nacional de Pesquisas Espaciais (INPE), a Universidade Estadual da Paraíba (UEPB), Universidade Federal de Campina Grande (UFCG), Universidade Federal de Alagoas (UFAL), Universidade Federal do Rio Grande do Norte (UFRN), Universidade Federal do Ceará (UFC), Universidade Federal de Pernambuco (UFPE), Universidade Federal Rural de Pernambuco (UFRPE), Universidade Federal do Rio de Janeiro (UFRJ), Universidade Federal de São Caros (UFSCar), Pontifícia Universidade Católica do Paraná (PUC-PR) e Universidade de São Paulo (USP), no Brasil.

Vale ressaltar que os projetos CAPES/Pró-equipamentos 2008, 2009 e 2010 aprovados sob a coordenação do PPGMDS foram integrados por vários Programas de Pós-Graduação, incluindo pesquisadores, doutorandos, mestrandos e alunos de iniciação científica. Esses projetos estão sendo executados e proporcionarão a todos os Programas melhorias na infraestrutura de pesquisa pelo acesso a novas tecnologias. No mesmo contexto, foram aprovados projetos FINEP/Pró-Infra em 2008, 2009 e 2010, igualmente sob coordenação do PPGMDS, envolvendo vários Programas de Pós-Graduação e grupos de pesquisa.

\section{Intercâmbios Internacionais}

Docentes do PPGMDS estão trabalhando em cooperação científica com as Universidades do Porto, Minho e Coimbra, de Portugal, com a YALE University dos EUA, com a Universidade de Alberta do Canadá e com a Université Toulouse III Paul Sabatier da França. Nestas duas últimas, duas discentes de doutorado realizaram sanduíches de seis meses, nos períodos de janeiro a julho de 2015 e de julho de 2015 a fevereiro de 2016, respectivamente. Na primeira, o projeto foi desenvolvido em conjunto com o Prof. Witold Pedrycz, sob o tema "Combinação de Métodos de Aglomeração Espacial Aplicados a Epidemiologia". Na segunda, o tema desenvolvido foi "Usabilidade de Interfaces para Serious Games".

Docentes do Programa realizaram visitas de trabalho científico a universidades estrangeiras em 
2015, a saber: Universidade de Orleans e Paris-Dauphine e Université Toulouse III Paul Sabatier, ambas na França e Universidade de Alberta, no Canadá.

Um docente do PPGMDS organizou um número especial da revista internacional "Knowledgebased Systems", ISSN: 0950-7051, em conjunto com o Prof. Luiz Martines da Universidade de Jaén, Espanha, sob o tema "Computational Intelligence Applications for Data Science". O número foi publicado em outubro de 2015, sob o volume 87 e está disponível no sítio: http://www. sciencedirect.com/science/journal/09507051/87

No ano de 2014, docentes do PPGMDS organizaram dois eventos (um internacional e um nacional): o 11th International FLINS Conference on Decision Making and Soft Computing (FLINS2014; http://www.de.ufpb.br/ flins2014/) e o III CBSF - Terceiro Congresso Brasileiro de Sistemas Fuzzy (CBSF 2014; http://www.de.ufpb.br/ cbsf2014/). Ambos ocorreram simultaneamente na cidade de João Pessoa entre 17 e 20 de agosto.

Docentes do PPMGDS atuam como membros de comitês de programa em vários congressos nacionais e internacionais, como "Congresso Brasileiro de Inteligência Computacional (CBIC 2012)", o "10th International Conference on Intelligent Systems and Knowledge Engineering (ISKE 2015)", “3rd Experiment@International Conference (Exp.at-2015)”, o“18th Symposium on Virtual and Augmented Reality (SVR 2016)" e o "12th International FLINS Conference on Uncertainty Modeling in Knowledge Engineering and Decision Making (FLINS 2016)". Os docentes do PPGMDS também tem servido como revisores de periódicos nacionais e internacionais como "Acta Amazônica", "Revista Latino America de Enfermagem", "Knowledge-Based Systems", "IEEE Transactions on Fuzzy Systems", "European Journal of Operational Research" e "Statistical Modelling", bem como integrantes de Corpo Editorial de periódicos, como "SBC Journal on 3D Interactive Systems" e a "Revista Tempus: Actas de Saúde Coletiva".

Somado a isso, o Programa tem conseguido executar o planejamento para vários dos seus docentes realizarem pós-doutorado e visitas a grupos de pesquisa no Brasil e exterior. Os primeiros discentes de doutorado realizaram sanduíches no exterior no ano de 2015, impulsionando e estreitando essas parcerias internacionais. Espera-se poder dar continuidade a esse fluxo de docentes e discentes em intercâmbio com instituições de excelência no exterior.

\section{Inserção Social}

A proposta do PPGMDS é atuar em problemas reais da sociedade, seja a partir de demandas de setores específicos, como as Secretarias de Saúde, ou a partir dos desdobramentos dos projetos de pesquisa desenvolvidos pelos docentes. Os resultados dos trabalhos são necessariamente a criação/ construção de um processo decisório que ajude na gestão dos problemas de saúde abordados. Neste sentido merece destaque alguns projetos e a descrição de seus objetivos e impactos esperados.

- A FiSIOTERAPIA E A PROMOÇÃO DA SÁUdE NA COMUNIDADE. Projeto de 
pesquisa/extensão, que contribui com a promoção da saúde dos moradores da comunidade do Grotão em João Pessoa-PB, através de ações educativas coletivas e do incentivo à participação popular. Este projeto envolve estudantes de graduação e alunos de pós graduação integrando a formação pessoal e profissional, bem como o empoderamento da comunidade.

- A INTERDISCIPLINARIDADE NA FORMAÇÃO E NO TRABALHO EM SAÚdE. objetiva analisar as possibilidades e limites de realização de ações interdisciplinares na formação em saúde e na prática profissional nos serviços de saúde.

- A LINHA DE CUIDAdO EM SAÚdE BUCAL NA 1a. MACRORREGIONAL DE SAÚDE DO ESTADO DA PARAÍBA. Projeto Integrado Financiado pelo CNPq Edital Saúde Bucal (Processo 3220080) com a participação de 32 pesquisadores, entre docentes e discentes do PPGMDS, docentes de outros departamentos e Profissionais de Saúde, com cobertura do serviço de saúde bucal de todo estado da Paraíba. Projeto ação que compatibiliza a pesquisa com a educação permanente em saúde, visando melhoria das práticas de cuidado.

- $\quad$ AVALIAÇÃO DO DESEMPENHO DE SERVIÇOS DE ATENÇÃO À SAÚDE MENTAL NA PARAÍBA. Tem a finalidade de difusão de novos conhecimentos produzidos na prática profissional, podendo contribuir também para a construção de novas possibilidades de atenção em saúde, tanto para as pessoas que apresentam transtornos psiquiátricos, como para as pessoas em situação de maior vulnerabilidade para o adoecimento de uma maneira geral, além de permitirà equipe de saúde/saúde mental um processo de trabalho articulado e com possibilidades de emancipação da opressão ideológica, característica do modelo de assistência tradicional contribuindo para a construção de um sistema local de saúde, mais acessível, integral e efetivo.

- $\quad$ AVALIAÇÃO DO IMPACTO DAS MUDANÇAS CLIMÁTICAS SOBRE AS DOENÇAS TRANSMITIDAS POR VETOR E INFECTO-CONTAGIOSAS NA PARAÍBA. O objetivo desse projeto consiste em pesquisar a influência das alterações bio-climáticas sobre a epidemiologia de doenças transmissíveis por vetores alados, bem como infecto-contagiosas, visando a vigilância e o controle das mesmas, para fornecer informações que subsidiem gestores e profissionais na elaboração e na aplicação de políticas públicas de saúde, respectivamente.

- AVALIAÇÃO DOS SERVIÇOS DE ATENÇÃO À SAÚdE DA MULHER. Objetiva comparar o planejamento e a formulação política dos programas de atenção à saúde da mulher com as práticas profissionais nos serviços públicos, no município de João Pessoa - PB.

- JOGOS EDUCACIONAIS MULTIPLATAFORMA. Pesquisa e desenvolvimento de jogos educacionais inteligentes para ensino fundamental usando técnicas de realidade virtual de baixo custo e telefonia celular. Os jogos devem conter algum tipo de sistemas inteligentes, que controlará os níveis do jogo e as suas diferentes fases, baseando-se em modelos de inteligência estatísticos, de crença, "fuzzy" e híbridos 
- AVALIAÇÃO DE HABILIDADES EM TREINAMENTOS REALIZADOS EM SIMULADORES BASEADOS EM REALIDADE VIRTUAL. Propõe a pesquisa de novas técnicas para a avaliação online de treinamentos realizados em ambientes de realidade virtual, baseados na generalização das redes probabilistas através da generalização da família de redes probabilistas bayesianas usando a teoria dos conjuntos fuzzy, a partir das suas distribuições estatísticas fuzzificadas.

\section{- INTELIGÊNCIA COMPUTACIONAL PARA APLICAÇÕES A PROBLEMAS COMPLEXOS NA ÁREA DE SAÚDE}

- $\quad$, que tem por objetivo ampliar a fronteira do conhecimento da Inteligência Computacional através da proposição de técnicas inovadoras baseadas nas Teorias dos Conjuntos Fuzzy, de Classificação/Reconhecimento de Padrões e da Estatística Computacional para modelagem, processamento e análise de dados e sinais na área de saúde. As principais aplicações das novas técnicas visam o projeto de classificadores, epidemiologia espacial, sistemas inteligentes de suporte à decisão e avaliação de treinamento psicomotor.

\section{- $\quad$ FATORES ASSOCIADOS E CONSEQUENCIAS DA INSEGURANÇA ALIMENTAR E} NUTRICIONAL. Identificar e analisar os fatores associados e as consequências da insegurança alimentar e nutricional na população paraibana, em particular das famílias residentes na região metropolitana de João Pessoa. Desta forma testar a relação de causalidade entre importantes indicadores de saúde materno infantil, como estado nutricional de mães e filhos, desenvolvimento cognitivo e comportamental infantil, morbidades da infância, práticas alimentares, stress, ansiedade e depressão maternos e conhecer as estratégias de sobrevivência e enfrentamento das situações adversas feitas pelas mães e o impacto dos programas de saúde voltados para este público com vistas a elaboração de políticas e programas de intervenção em saúde.

\section{- NOVAS TECNOLOGIAS PARA IMERSÃO E COLABORAÇÃO HÁPTICA EM} TREINAMENTO A DISTÂNCIA PARA MEDICINA. Insere-se no contexto das novas tecnologias para a medicina colaborativa com suporte a sistemas hápticos, visando pesquisar formas de otimizar o processo de colaboração háptica de modo a permitir seu uso em redes convencionais (Internet) e plataformas de baixo custo. Espera-se com isto, contribuir para a popularização desta tecnologia para o ensino médico, aproximando especialistas e alunos, bem como permitir a oferta de formas mais seguras, éticas e com menor custo de treinamento.

- $\quad$ TOMADA DE DECISÃO NAS UNIDADES BÁSICAS DE SAÚDE. A finalidade desta pesquisa é refletir sobre a Tomada de Decisão dos enfermeiros nas Unidades Básicas de Saúde, buscando ampliar o arsenal de ferramentas administrativas disponíveis para estes profissionais na execução de atividades gerenciais. 
- MAPEAMENTO DOS ATOS E AÇOES DE SAÚDE OFERTADAS NAS UNIDADES DA ESTRATÉGIA SAÚDE DA FAMÍLIA E AS INTERNAÇÕES POR CONDIÇÕES SENSÍVEIS À ATENÇÃO PRIMÁRIA. Baseando-se no método de análise de componentes principais, a partir da matriz de covariância dos dados para fundamentar a construção de um indicador estatístico sintético visando o ranqueamento dos municípios quanto as Internações por condições sensíveis a atenção primária.

Estes projetos têm foco e campo de atuação nas práticas dos serviços de saúde e muitas vezes participação de atores sociais importantes para a melhoria da atenção e do cuidado à saúde da população.

\section{Interfaces com a Educação Básica}

No contexto das pesquisas de metodologias inteligentes para avaliação, pesquisadores do PPGMDS tem desenvolvido e testado metodologias de modo a dotar sistemas computacionais voltados ao treinamento, capacitação e educação. Algumas destas metodologias foram adequadas e implementadas em jogos voltados para a educação fundamental, particularmente nas disciplinas de matemática e geografia. Está em desenvolvimento um jogo para apoio ao ensino de Biologia que deve estar finalizado no segundo semestre de 2016.

Deste modo, as metodologias foram inseridas como módulos de inteligência artificial em jogos que estão disponíveis para download gratuito e que podem ser executados em dispositivos móveis e computadores pessoais. Tem-se conhecimento do uso destes jogos por escolas de João Pessoa/ PB e do sul do país.

Em um contexto profissionalizante, outros 3 jogos foram desenvolvidos para capacitação e educação continuada. Um deles voltado ao aprendizado de técnicas de escovação bucal, outro ao treinamento de equipes para a realização de procedimentos cirúrgicos e, mais recentemente tem sido testado na rede municipal de João Pessoa um jogo voltado à capacitação de profissionais da saúde quanto à violência contra a mulher.

\section{Produção científica}

O grupo tem trabalhado para ampliar a produção científica para os estratos mais altos do QualisCAPES, objetivando alcançar a meta de no mínimo 70\%. Em 2012, o percentual de artigos nos estratos de A1 a B2 foi de 39,29\%. No ano de 2013, esse percentual subiu para $73,24 \%$ e em 2014 aumentou para 86,11\%. Em 2015 esse percentual ficou 74,24\%, portanto dentro da meta programada.

O PPGMDS tem trabalhado para e manter um alto número de publicações anuais por docente. Em 2012, essa média foi de 1,57 artigo/docente, em 2013 foi de 3,55 artigos/docente, em 2014 foi de 3,60 artigos/docente e em 2015 foi de 3,00 artigos/docente. Paralelamente, o Programa 
tem obtido sucesso na manutenção de um alto número de artigos publicados em periódicos com participação de discentes do Programa. Em 2012 foram 17 artigos conjuntos; em 2013 ,16 artigos, em 2014, 22 artigos e em 2015, 23 artigos tiveram discentes como co-autores.

O fortalecimento da inserção social do PPGMDS na comunidade da saúde tem sido trabalhado. Docentes do PPGMDS tem atuado sobre problemas reais da sociedade, através de demandas das Secretarias municipais e estadual. Outro fator a ser destacado é a disponibilização de tecnologias desenvolvidas por docentes e discentes do Programa, como por exemplo: jogos para aprendizado de técnicas de escovação bucal, treinamento de equipes para a realização de procedimentos cirúrgicos e capacitação de profissionais da saúde quanto à violência contra a mulher).

\section{REFERÊNCIAS BIBLIOGRÁFICAS}

Programa de Pós-graduação em Modelos de Decisão e Saúde. Diponível em http://sistemas. ufpb.br/sigaa/public/programa/portal.jsf?id=1895 Acesso em: 22 jun de 2016.

D.O.U. Diário Oficil da União. Portaria de Reconhecimento do Programa de Pós-graduação em Modelos de Decisão e Saúde. Disponível em http://www.de.ufpb.br/ mds/DiarioOficialReconhece PPGMDS.pdf Acesso em: 22 jun de 2016.

RESOLUÇÃO No 02/2012 Disciplina o Processo de Matrícula para os discentes do Programa de Pós-Graduação em Modelos de Decisão e Saúde do Centro de Ciências Exatas e da Natureza da UFPB. Disponível em: http://www.de.ufpb.br/ mds/Resolucao_PPGMDS 02-2012_Disciplina Matricula_PPGMDS.pdf Acesso em: 22 jun de 2016.

RESOLUÇÃO No 01/2013. Disciplina as normas do estágio docência dos alunos do Programa de Pós-Graduação em Modelos de Decisão e Saúde do Centro de Ciências Exatas e da Natureza da UFPB. Disponível em: http://sistemas.ufpb.br/sigaa/verProducao?idProducao=110364\&key=359

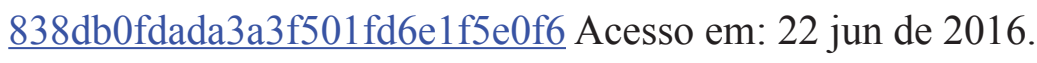

BRASIL. Lei n. 9394, de 20 de dezembro de 1996. Estabelece as Diretrizes e Bases da Educação Nacional. Brasília (DF): Senado; 1996

Artigo apresentado em 24/05/16

Artigo aprovado em 20/06/16 Artigo publicado no sistema em 30/06/16 